\title{
CONTINUITY PROPERTIES OF ATTRACTORS FOR ITERATED FUZZY SET SYSTEMS
}

\author{
B. FORTE ${ }^{1}$, M. LO SCHIAVO ${ }^{2}$ and E. R. VRSCAY ${ }^{1}$
}

(Received 2 November 1992; revised 1 March 1993)

\begin{abstract}
An $N$-map Iterated Fuzzy Set System (IFZS), introduced in [4] and to be denoted as (w, $\Phi$ ), is a system of $N$ contraction maps $w_{i}: X \rightarrow X$ over a compact metric space $(X, d)$, with associated "grey level" maps $\phi_{i}:[0,1] \rightarrow[0,1]$. Associated with an IFZS $(w, \Phi)$ is a fixed point $u \in \mathscr{F}^{*}(X)$, the class of normalized fuzzy sets on $X, u: X \rightarrow[0,1]$. We are concemed with the continuity properties of $u$ with respect to changes in the $w_{i}$ and the $\phi_{i}$. Establishing continuity for the fixed points of IFZS is more complicated than for traditional Iterated Function Systems (IFS) with probabilities since a composition of functions is involved. Continuity at each specific attractor $u$ may be established over a suitably restricted domain of $\phi_{i}$ maps. Two applications are (i) animation of images and (ii) the inverse problem of fractal construction.
\end{abstract}

\section{Introduction}

We consider a special class of dynamical systems over function spaces which has been introduced in [4] and referred to as Iterated Fuzzy Set Systems (IFZS). An IFZS, to be denoted as $(\mathbf{w}, \Phi)$, is a system $\mathbf{w}$ of $N$ contraction mappings $w_{i}(x): X \rightarrow X$ over a compact metric space $(X, d)$ (the "base space"), with an associated system $\Phi$ of mappings $\phi_{i}:[0,1] \rightarrow[0,1]$ (the "grey level maps"). The IFZS incorporates, at least in part, the idea of set-valued contraction mappings over a compact metric space, developed by Hutchinson [9] and Barnsley and coworkers [1]-[3], the latter referring to such systems as Iterated Function Systems (IFS). There is, however, a fundamental difference between the IFS and the IFZS. The IFS works with measures and a set of probabilities $p_{i}$ associated with the $w_{i}$ which act as multiplicative weights. The IFZS works with functions $u: X \rightarrow[0,1]$ and functions $\phi_{i}:[0,1] \rightarrow[0,1]$ which are composed with the $u$. From the viewpoint of image processing or pattern recognition,

\footnotetext{
${ }^{1}$ Department of Applied Mathematics, University of Waterloo, Waterloo, Canada N2L 3G1.

${ }^{2}$ Metodi e Mod. Mat. Sc. Appl., Universita' di Roma 'La Sapienza”, 00161 Rome, Italy.

(C) Australian Mathematical Society, 1994, Serial-fee code 0334-2700/94
} 
the value $u(x)$ may be interpreted as a nonnegative grey level or brightness value at the point (or pixel) $x \in X$. The IFS component of the IFZS, a set-valued mapping $\mathbf{w}$ which acts on nonempty compact subsets of $X$, will operate on the level sets of $u$. As such, it is natural to consider $u \in \mathscr{F}(X)$, the class of fuzzy sets on $X$; compactness of the level sets requires that the $u$ be restricted to the subspace $\mathscr{F}^{*}(X) \subset \mathscr{F}(X)$ of (normalized) upper semicontinuous fuzzy sets with the $d_{\infty}$ metric [7] involving Hausdorff distances between their level sets. (In the literature, $\mathscr{F}^{*}(X)$ continues to be referred to as the class of fuzzy sets on $X$ because of its historical probabilistic interpretations.) Associated with each IFZS $(\mathbf{w}, \Phi)$ is a contractive operator $T$ which possesses a unique fixed point $u$, the attractor of the IFZS. The support of $u$ is a subset of the attractor $A \subseteq X$ of the IFS defined by $w$. The relevance of the IFZS approach to image processing has been discussed in [4]. In addition, our preliminary studies indicate that the IFZS affords a considerable simplification in the treatment of the inverse problem of fractal or image construction [1]-[5], [10], [11].

From the viewpoint of image representation, an important advantage of an IFStype method over function spaces (in this paper, $\mathscr{F}^{*}(X)$ with the $d_{\infty}$-metric) is that it permits a more direct control of grey level or brightness values at individual points $x \in X$. This has been realized in more recent "fractal compression schemes" [2], [10]. In another paper, a complete connection between the IFZS method and these schemes will be provided [8].

In this paper, we are concerned specifically with the continuity properties of invariant sets $u$ for $N$-map IFZS, particularly with respect to changes in the contraction maps $w_{i}$ and the grey level maps $\phi_{i}$. Barnsley [1] has shown that the attractor of a contractive IFS varies continuously with respect to parameters in the IFS maps. An obvious application is "animation", where IFS attractors representing images are deformed in an apparently continuous manner in time. As well, continuity results are clearly important for the inverse problem, where local "fine tuning" of the IFS maps and probabilities is performed to optimize the approximation of sets or measures with IFS attractors or invariant measures. In [6], the (uniform) continuity of IFS attractors and invariant measures with respect to arbitrary variations in the IFS maps and/or associated probabilities was derived, a generalization of the results in [1]. The continuity followed naturally from the rather simple structure of IFS: the metrics employed for the map vectors $\mathbf{w}$ and the probability vectors $\mathbf{p}$ are easily and directly related to (i) the Hausdorff metric $h$ on $\mathscr{H}(X)$, the set of nonempty compact subsets of $X$ and (ii) the Hutchinson metric $d_{H}$ on $\mathscr{M}(X)$, the set of probability measures on $\mathscr{B}(X)$, the $\sigma$-algebra of Borel subsets of $X$.

Establishing continuity of IFZS attractors with respect to IFS or grey level maps is more complicated, primarily because a composition of the $\phi$ and $u$ functions is involved. From the Banach fixed point theorem, it easily follows that the attractor $u$ is continuous with respect to the IFZS operator $T$. However, the $T$ operator is not 
continuous with respect to the grey level maps $\phi_{i}$. Thus it is not possible to use the $T$ operator (unlike the IFS case) to prove that $u$ is continuous with respect to the $\phi_{i}$ maps. A main result of this paper is that we may bypass the use of the $T$ operator and establish a "local" continuity of $u$ with respect to the $\phi_{i}$ and $w_{i}$ maps. Uniform continuity is sacrificed, but this is not a great price, since a continuous variation in $u$ can still be produced by means of a controlled variation of the $\phi_{i}$.

The layout of this paper is as follows. In Section 2, some basic properties of IFZS are presented in terms of the IFS and grey-level map components. In Section 3 we derive a basic continuity property of fixed points of contractive maps. We then review the "well-behaved" continuity properties of IFS attractors and invariant measures. The rather trivial continuity property of an IFZS fixed point with respect to the IFS maps $w_{i}$ is then shown. Finally, we show that the contractive $T$ operator of an IFZS is discontinuous with respect to the grey level maps $\phi_{i}$. In Section 4 , we define an appropriate "local" distance function for the $\phi_{i}$ maps and derive the continuity of $u$ with respect to a restricted set of $\phi_{i}$. Section 5 is devoted to a further discussion of these results and suggestions for future research. Appendix A contains a brief discussion of invariant measures for IFS to help the general reader understand the IFS continuity results outlined in Section 3.

\section{Preliminaries: Iterated Fuzzy Set Systems}

In this section we introduce all the relevant notation and outline the salient features of IFZS. The reader is referred to [4] for details. An $N$-map IFZS, denoted as (w, $\Phi$ ), has an IFS component, $w$, on a base space $(X, d)$ and a grey-level component, $\Phi$, on $[0,1]$, as outlined below.

A. The IFS component: As above, $(X, d)$ will denote a compact metric space. Let $\mathbf{w}$ denote a set of $N$ contraction maps on $X$, that is, $\mathbf{w}=\left(w_{1}, w_{2}, \ldots, w_{N}\right), w_{i}: X \rightarrow X$ such that for all $x, y \in X$,

$$
d\left(w_{i}(x), w_{i}(y)\right) \leq s_{i} d(x, y), \quad 0 \leq s_{i}<1, \quad i=1,2, \ldots, N .
$$

We shall refer to $s_{i}$ as the contractivity factor of the map $w_{i}$ : the contractivity factor of the IFS $w$ is defined as $s=\max _{1 \leq i \leq N} s_{i}$.

Let $\mathscr{H}(X)$ denote the set of nonempty compact subsets of $X$. The Hausdorff distance between two sets $A, B \in \mathscr{H}(X)$ is given by

$$
h(A, B) \equiv \max \{D(A, B), D(B, A)\},
$$

where

$$
D(A, B)=\sup _{x \in A} \inf _{y \in B} d(x, y) .
$$


$(\mathscr{H}(X), h)$ is a complete metric space.

Given an IFS $\mathbf{w}$, define the set-valued mapping $\hat{\mathbf{w}}: \mathscr{H}(X) \rightarrow \mathscr{H}(X)$ as follows:

$$
\hat{\mathbf{w}}(S)=\bigcup_{i=1}^{N} w_{i}(S), \quad S \in \mathscr{H}(X),
$$

where $w_{i}(S) \equiv\left\{w_{i}(x), x \in S\right\}$. As is well known [1,9], there exists a unique set $A \in \mathscr{H}(X)$, the attractor of the IFS, which is invariant with respect to $\hat{w}$, that is,

$$
A=\hat{\mathbf{w}}(A)=\bigcup_{i=1}^{N} w_{i}(A)
$$

This result follows from the fact that $\hat{\mathbf{w}}$ is a contraction mapping on $(\mathscr{H}(X), h)$ : for $A, B \in \mathscr{H}(X)$,

$$
h(\hat{\mathbf{w}}(A), \hat{\mathbf{w}}(B)) \leq \operatorname{sh}(A, B) .
$$

As well, from the contraction mapping principle,

$$
h\left(\hat{\mathbf{w}}^{n}(S), A\right) \rightarrow 0 \quad \text { as } n \rightarrow \infty, \quad \forall S \in \mathscr{H}(X)
$$

SIMPLE EXAMPLES. Let $X=[0,1]$ and $N=2$, with $w_{1}(x)=s x, w_{2}(x)=s x+(1-$ $s)$. (i) For $s \geq 1 / 2, A=[0,1]$. (ii) For $s=0, A=\{0,1\}$. (iii) For $0<s<1 / 2, A$ is a Cantor-like set on $[0,1]$. When $s=1 / 3, A$ is the ternary Cantor set on $[0,1]$.

B. The grey level component: Let $\mathscr{F}(X)$ denote the class of functions $u: X \rightarrow$ $[0,1]$. We consider the following subclass of functions [4], $\mathscr{F}^{*}(X) \subset \mathscr{F}(X): u \in$ $\mathscr{F}^{*}(X)$ if and only if

(i) $u \in \mathscr{F}(X)$,

(ii) $u$ is upper semicontinuous on $(X, d)$,

(iii) $u$ is normalized, i.e., $u\left(x_{0}\right)=1$ for some $x_{0} \in X$.

Now define the $\alpha$-level set, $[u]^{\alpha}$, of $u \in \mathscr{F}^{*}(X)$ as follows:

$[u]^{\alpha}=\{x \in X: u(x) \geq \alpha\}$, for $0<\alpha \leq 1$,

$[u]^{0}=\overline{\{x \in X: u(x)>0\}}$ (the bar denotes closure).

From properties (ii) and (iii) above, the metric space $(\mathscr{H}(X), h)$ contains all $\alpha$-level sets, $0 \leq \alpha \leq 1$, of all $u \in \mathscr{F}^{*}(X)$. We consider the following metric on $\mathscr{F}^{*}(X)$ :

$$
d_{\infty}(u, v)=\sup _{0 \leq \alpha \leq 1}\left\{h\left([u]^{\alpha},[v]^{\alpha}\right)\right\}, \quad \forall u, v \in \mathscr{F}^{*}(X)
$$

The metric space $\left(\mathscr{F}^{*}(X), d_{\infty}\right)$ is complete [7]. 
Let $\mathscr{M}^{+}([0,1])$ denote the set of all functions $\phi:[0,1] \rightarrow[0,1]$ with the following properties:

(i) $\phi$ is nondecreasing on $[0,1]$,

(ii) $\phi$ is right continuous on $[0,1)$,

(iii) $\phi(0)=0$,

(iv) $\phi\left(t^{*}\right)>0$ for at least one $t^{*} \in(0,1]$.

Now given an IFS w, let $\Phi=\left(\phi_{1}, \phi_{2}, \ldots, \phi_{N}\right)$ denote a set of associated grey level maps which satisfy the following conditions:

(a) $\phi_{i} \in \mathscr{M}^{+}([0,1])$ for $i \in\{1,2, \ldots, N\}$,

(b) $\phi_{i^{*}}(1)=1$ for at least one $i^{*} \in\{1,2, \ldots, N\}$.
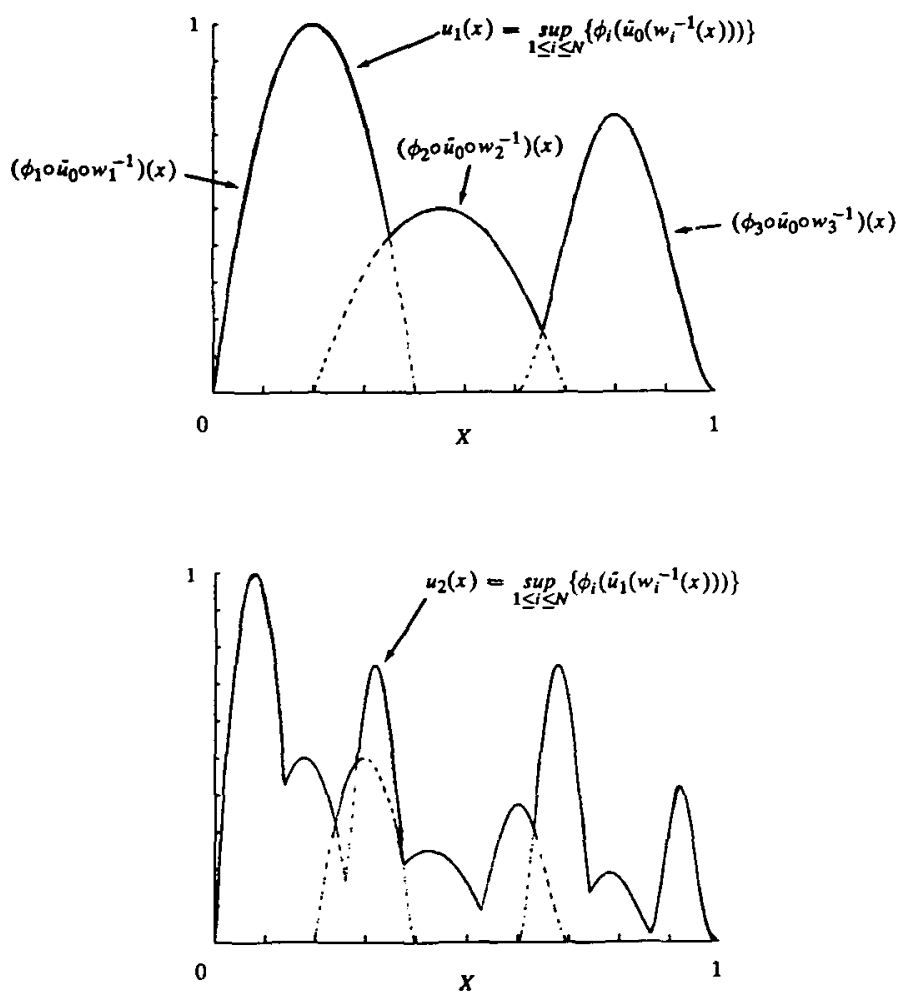

FIGURE 1. Graphs of $u_{1}(x)=\left(T u_{0}\right)(x)$ and $u_{2}(x)=\left(T u_{1}\right)(x)$, where $u_{0}(x)=4 x(1-x)$ and $T$ is the contractive operator for the following three-map IFZS on $X=[0,1]$ :

$$
\begin{array}{ll}
w_{1}(x)=0.4 x, & \phi_{1}(t)=t \\
w_{2}(x)=0.5 x+0.2, & \phi_{2}(t)=0.5 t, \\
w_{3}(x)=0.4 x+0.6, & \phi_{3}(t)=0.75 t^{2} .
\end{array}
$$


The pair of vectors (w, $\Phi)$ constitutes an $N$-map IFZS. Properties (i), (ii) and (b) will guarantee that the contractive operator $T$, to be defined below, will map $\mathscr{F}^{*}(X)$ into itself. Property (iii) is a natural assumption in the consideration of grey level functions: if the grey level of a point (or pixel) $x \in X$ is zero, then it should remain zero after being acted upon by the $\phi_{i}$ maps. Property (iv) will be explained below.

We now introduce the following operator ${ }^{3} T: \mathscr{F}^{*}(X) \rightarrow \mathscr{F}^{*}(X)$ :

$$
(T u)(x)=\sup _{1 \leq i \leq N}\left\{\phi_{i}\left(\tilde{u}\left(w_{i}^{-1}(x)\right)\right)\right\},
$$

where, for $B \subset X$,

$$
\begin{aligned}
\tilde{u}(B) & :=\sup _{y \in B}\{u(y)\}, \quad \text { if } B \neq \emptyset, \\
\tilde{u}(\emptyset) & :=0 .
\end{aligned}
$$

The action of the operator $T$ for a simple IFZS on $[0,1]$ is illustrated in Figure 1.

In [4] it was proved that $T$ is a contraction mapping on the space $\left(\mathscr{F}^{*}(X), d_{\infty}\right)$ :

$$
d_{\infty}\left(T u_{1}, T u_{2}\right) \leq s d_{\infty}\left(u_{1}, u_{2}\right) \quad \forall u_{1}, u_{2} \in \mathscr{F}^{*}(X) .
$$

Thus, there exists a unique function $u \in \mathscr{F}^{*}(X)$, the attractor of the IFZS, such that

$$
T u=u .
$$

This, in turn, implies that $u$ is the unique solution to the following functional equation in the unknown $v \in \mathscr{F}^{*}(X)$,

$$
v(x)=\sup \left\{\phi_{1}\left(\tilde{v}\left(w_{1}^{-1}(x)\right)\right), \phi_{2}\left(\tilde{v}\left(w_{2}^{-1}(x)\right)\right), \ldots, \phi_{N}\left(\tilde{v}\left(w_{N}^{-1}(x)\right)\right)\right\}, \quad \forall x \in X .
$$

The attractor $u$ for the IFZS of Figure 1 is sketched in Figure 2.

Let $\mathscr{T}_{N}$ denote the set of all contractive operators $T$ corresponding to $N$-map IFZS on $(X, d)$. A noteworthy property of these operators is the following: for $v \in \mathscr{F}^{*}(X)$,

$$
[T v]^{\alpha}=\bigcup_{i=1}^{N} w_{i}\left(\left[\phi_{i} \circ v\right]^{\alpha}\right), \quad 0 \leq \alpha \leq 1 .
$$

Thus, when $v=u=T u$,

$$
[u]^{\alpha}=\bigcup_{i=1}^{N} w_{i}\left(\left[\phi_{i} \circ u\right]^{\alpha}\right), \quad 0 \leq \alpha \leq 1,
$$

which is a generalized self-tiling property of $\alpha$-level sets for IFZS attractors.

\footnotetext{
${ }^{3}$ Note: In [4], this operator was denoted $T_{s}$ in order to emphasize the fact that the supremum function was chosen as the associative operator on $\mathscr{F}^{*}(X)$. For notational convenience, we have dropped the subscript $s$ in this paper.
} 


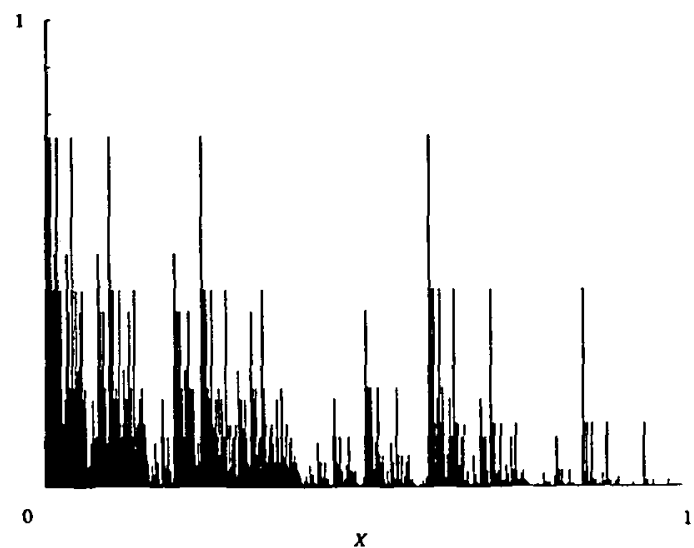

FIGURE 2. Histogram approximation of the attractor $u=T u$ for the IFZS (w, $\phi$ ) of Figure 1 . Note that $u(0)=1$.

\section{Continuity of fixed points and problems with the $d_{C o n\left(\mathscr{F}^{*}(X)\right)}$ metric}

Let $\left(Y, d_{Y}\right)$ be a complete metric space and denote by

$$
\begin{array}{r}
\operatorname{Con}(Y)=\left\{f: Y \rightarrow Y \mid \exists s_{f} \in[0,1): d_{Y}(f(x), f(y)) \leq s_{f} d_{Y}(x, y),\right. \\
\forall x, y \in Y\},
\end{array}
$$

a set of contraction mappings on $\left(Y, d_{Y}\right)$. Now consider the following natural metric on this function space:

$$
d_{C o n(Y)}(f, g) \equiv \sup _{y \in Y} d_{Y}(f(y), g(y)), \quad f, g \in \operatorname{Con}(Y) .
$$

Note that the metric space ( $\left.\operatorname{Con}(Y), d_{\operatorname{Con}(Y)}\right)$ itself may not be complete. Now let $y_{f}$ and $y_{g}$ be the unique fixed points of the maps $f$ and $g$, respectively, that is, $f\left(y_{f}\right)=y_{f}$ and $g\left(y_{8}\right)=y_{g}$.

PROPOSITION 3.1. The following inequality holds:

$$
d_{Y}\left(y_{f}, y_{g}\right)<\frac{1}{1-s_{f}} d_{C o n(Y)}(f, g),
$$

where $s_{f}$ is the contractivity factor of $f$.

PROOF.

$$
\begin{aligned}
d_{Y}\left(y_{f}, y_{g}\right) & =d_{Y}\left(f\left(y_{f}\right), g\left(y_{g}\right)\right) \\
& \leq d_{Y}\left(f\left(y_{f}\right), f\left(y_{g}\right)\right)+d_{Y}\left(f\left(y_{g}\right), g\left(y_{g}\right)\right) \\
& <s_{f} d\left(y_{f}, y_{g}\right)+d_{C o n(Y)}(f, g),
\end{aligned}
$$

which then yields (3.3). 
Inequality (3.3) implies continuity for the fixed point $y_{f}$ with respect to $f$, uniformly on the set of contractive maps in $\left(\operatorname{Con}(Y), d_{\operatorname{Con}(Y)}\right)$ with contractivity factors $0 \leq s_{f} \leq$ $s<1$. Such continuity would be useless when the topology induced on $\operatorname{Con}(Y)$ by $d_{\operatorname{Con}(Y)}$ is such that every element $f \in \operatorname{Con}(Y)$ is isolated, that is, there exists an $\epsilon>0$ such that $N_{\epsilon}(f) \backslash\{f\}=\emptyset$, where $N_{\epsilon}(f)=\left\{g \in \operatorname{Con}(Y): d_{C o n(Y)}(f, g)<\epsilon\right\}$ denotes the $\epsilon$-neighbourhood of $f$. This is, in fact, the situation for IFZS attractors as we show below. However, in order that the reader may well appreciate the difficulties posed by the IFZS problem, we first review the basic "well-behaved" continuity results for "traditional" IFS attractors and measures.

\subsection{Continuity results for "traditional" IFS attractors and invariant measures}

The basic features of IFS with probabilities (IFSP) and their invariant measures are given in Appendix A. In the following discussion, it will be convenient to consider sequences of contractive IFSP, $\left(\mathbf{w}_{k}, \mathbf{p}_{k}\right), k=1,2, \ldots$, where

$$
\mathbf{w}_{k}=\left(w_{k 1}, w_{k 2}, \ldots, w_{k N}\right), \quad \mathbf{p}_{k}=\left(p_{k 1}, p_{k 2}, \ldots, p_{k N}\right) .
$$

Let $s_{k j}$ denote the contractivity factors of the $w_{k j}$; the contractivity factor of the IFS $\mathbf{w}_{k}$ is then $s_{k}=\max _{1 \leq j \leq N} s_{k j}$. We then define the following natural metrics for the map and probability vectors, respectively,

$$
\begin{aligned}
d_{w}^{N}\left(\mathbf{w}_{1}, \mathbf{w}_{2}\right) & \equiv \max _{1 \leq i \leq N} \sup _{x \in X} d\left(w_{1 i}(x), w_{2 i}(x)\right) . \\
d_{p}^{N}\left(\mathbf{p}_{1}, \mathbf{p}_{2}\right) & \equiv \max _{1 \leq i \leq N}\left|p_{1 i}-p_{2 i}\right| .
\end{aligned}
$$

Using these metrics, the following continuity results were derived in [6].

PROPOSITION 3.2. Let $\mathbf{w}_{1}$ be an $N$-map contractive IFS with contractivity factor $s_{1}$ and attractor $A_{1}$. Then for any $\epsilon>0$ there exists $a \delta>0$ such that for all $N$-map IFS $\mathbf{w}_{2}$ satisfying $d_{w}^{N}\left(\mathbf{w}_{1}, \mathbf{w}_{2}\right)<\delta$, it follows that $h\left(A_{1}, A_{2}\right)<\epsilon$, where $A_{2}$ is the attractor of $\mathbf{w}_{2}$.

PROPOSITION 3.3. Let $\left(\mathbf{w}_{1}, \mathbf{p}_{1}\right)$ be an $N$-map contractive IFS with probabilities, with contractivity factor $s_{1}$ and invariant measure $\mu_{1}$. Then for every $\epsilon>0$, there exist $\delta_{1}, \delta_{2}>0$ such that for all $N$-map IFSP $\left(\mathbf{w}_{2}, \mathbf{p}_{2}\right)$ satisfying

$$
d_{w}^{N}\left(\mathbf{w}_{1}, \mathbf{w}_{2}\right)<\delta_{1}, \quad d_{p}^{N}\left(\mathbf{p}_{1}, \mathbf{p}_{2}\right)<\delta_{2},
$$

it follows that $d_{H}\left(\mu_{1}, \mu_{2}\right)<\epsilon$, where $\mu_{2}$ is the invariant measure of the $\operatorname{IFSP}\left(\mathbf{w}_{2}, \mathbf{p}_{2}\right)$.

Proposition 3.2, which follows immediately from Proposition 3.1 with $\delta=\epsilon(1-$ $s_{1}$ ), is a generalization of Barnsley's result [1, Sect. 3.11] showing continuity of the attractor $A$ with respect to a parameter in the IFS maps. As for the proof of 
Proposition 3.3, let $Y=\mathscr{M}(X)$ (see Appendix A) and let $\operatorname{Con}(Y)$ be the space of Markov operators corresponding to all contractive $N$-map IFS with probabilities. Then define the following metric on this space:

$$
d_{M}^{N}\left(M_{1}, M_{2}\right) \equiv \sup _{\mu \in \mathscr{M}(X)} d_{H}\left(M_{1} \mu, M_{2} \mu\right)
$$

After suitably defining the $\delta_{i}$, the result then follows from Proposition 3.1.

3.2. Continuity results for IFZS attractors Now let $\left(Y, d_{Y}\right)=\left(\mathscr{F}^{*}(X), d_{\infty}\right)$ and $\operatorname{Con}\left(\mathscr{F}^{*}(X)\right)=\mathscr{T}_{N}$, the space of contractive $N$-map IFZS operators on $\left(\mathscr{F}^{*}(X), d_{\infty}\right)$. It will be convenient to index the IFZS operators as $T_{k} \in \mathscr{T}_{N}, k=1,2,3, \ldots$, with action defined by

$$
\left(T_{k} u\right)(x)=\sup _{1 \leq i \leq N}\left\{\phi_{k i}\left(\tilde{u}\left(w_{k i}^{-1}(x)\right)\right)\right\}, \quad u \in \mathscr{F}^{*}(X),
$$

where the corresponding IFZS has been denoted as $\left(\mathbf{w}_{k}, \Phi_{k}\right)$,

$$
\mathbf{w}_{k}=\left(w_{k 1}, w_{k 2}, \ldots, w_{k N}\right), \quad \Phi_{k}=\left(\phi_{k 1}, \phi_{k 2}, \ldots, \phi_{k N}\right) .
$$

Also let $u_{k}$ denote the fuzzy set attractor of $\left(\mathbf{w}_{k}, \Phi_{k}\right)$, that is, $T_{k} u_{k}=u_{k}$. Note that if $T_{k} u=T_{l} u$ for all $u \in \mathscr{F}^{*}(X)$, then we consider $T_{k}$ to be equal to $T_{l}$. For example, the action of $T_{k}$ is unchanged when the $w_{k i}$ and associated $\phi_{k i}$ maps are permuted.

In analogy to the IFS case, cf. (3.7), it appears natural to choose the following distance function on $\operatorname{Con}\left(\mathscr{F}^{*}(X)\right)$ (see also [7]),

$$
d_{C o n\left(\mathscr{F}^{*}(X)\right)}\left(T_{k}, T_{l}\right)=\sup _{u \in \mathscr{F}^{*}(X)} d_{\infty}\left(T_{k} u, T_{l} u\right)
$$

which defines a metric on $\mathscr{T}_{N}$. (If $d_{\operatorname{Con}\left(\mathscr{F}^{*}(X)\right)}\left(T_{1}, T_{2}\right)=0$, then $d_{\infty}\left(u_{1}, u_{2}\right)=0$.) Continuity of the fixed points $u_{k}$ with respect to the $T_{k}$ operators follows immediate from Proposition 3.1. However, this property contains no information: as in the IFS case, it would be desirable to formulate continuity of the $u_{k}$ in terms of the IFZS components $\mathbf{w}_{k}$ and $\Phi_{k}$. The following result shows that continuity with respect to the IFS maps $w_{k i}$ is trivial.

PROPOSITION 3.4. Let $\left(\mathbf{w}_{1}, \Phi_{1}\right)$ be an IFZS with contractivity factor $s_{1}$, associated operator $T_{1} \in \mathscr{T}_{N}$ and attractor $u_{1} \in \mathscr{F}^{*}(X)$. Then for every $\epsilon>0$, there exists a $\delta>0$ such that for any IFZS $\left(\mathbf{w}_{2}, \Phi_{1}\right)$ satisfying $d_{w}^{N}\left(\mathbf{w}_{1}, \mathbf{w}_{2}\right)<\delta$, it follows that

$$
d_{\infty}\left(u_{1}, u_{2}\right)<\epsilon \text {, }
$$

where $u_{2} \in \mathscr{F}^{*}(X)$ is the attractor of the $\operatorname{IFZS}\left(\mathbf{w}_{2}, \Phi_{1}\right)$. 
Proof. Given an $\epsilon>0$, let $\mathbf{w}_{2}$ be such that

$$
d_{w}^{N}\left(\mathbf{w}_{1}, \mathbf{w}_{2}\right)<\epsilon\left(1-s_{1}\right) .
$$

Let $T_{2} \in \mathscr{T}_{N}$ be the operator associated with the IFZS $\left(\mathbf{w}_{2}, \Phi_{1}\right)$. Then

$$
\begin{aligned}
d_{\text {Con }\left(\mathscr{F}^{*}(X)\right)}\left(T_{1}, T_{2}\right) & =\sup _{u \in \mathscr{F}^{*}(X)} d_{\infty}\left(T_{1} u, T_{2} u\right) \\
& =\sup _{u \in \mathscr{F}^{*}(X)} \sup _{0 \leq \alpha \leq 1} h\left(\left[T_{1} u\right]^{\alpha},\left[T_{2} u\right]^{\alpha}\right) \\
& =\sup _{u \in \mathscr{F}^{*}(X)} \sup _{0 \leq \alpha \leq 1} h\left(\bigcup_{1 \leq i \leq N} w_{1 i}\left(\left[\phi_{1 i} \circ u\right]^{\alpha}\right), \operatorname{Suj}_{1 \leq j \leq N} w_{2 j}\left(\left[\phi_{1 j} \circ u\right]^{\alpha}\right)\right) \\
& \leq \sup _{u \in \mathscr{F}^{*}(X)} \sup _{0 \leq \alpha \leq 1} \max _{1 \leq i \leq N} h\left(w_{1 i}\left(\left[\phi_{1 i} \circ u\right]^{\alpha}\right), w_{2 i}\left(\left[\phi_{1 i} \circ u\right]^{\alpha}\right)\right) \\
& \leq d_{w}^{N}\left(\mathbf{w}_{1}, \mathbf{w}_{2}\right) \\
& <\epsilon\left(1-s_{1}\right) .
\end{aligned}
$$

Note that we have used the following property of the Hausdorff metric:

$$
h\left(\bigcup_{1 \leq i \leq N} A_{i}, \cup_{1 \leq j \leq N} B_{j}\right) \leq \max _{1 \leq i \leq N} h\left(A_{i}, B_{i}\right) .
$$

From Proposition 3.1, the desired result then follows.

In other words, with the grey-level maps fixed, the $T_{k}$ operators are continuous with respect to the IFS maps $w_{k i}$ in the $d_{C o n\left(\mathscr{F}^{*}(X)\right)}$ metric and continuity of the attractors is implied through Proposition 3.1. However, we now show that the $T_{k}$ are not necessarily continuous with respect to the grey level maps $\phi_{k i}$. We focus on particularly important subsets of $\operatorname{Con}\left(\mathscr{F}^{*}(X)\right)$, namely, those sections of $\mathscr{T}_{N}$ obtained by fixing the IFS maps $\mathbf{w}_{k}$ and varying the grey level maps $\phi_{k i}$ one at a time.

DEFINITION 3.1. Let $(\mathbf{w}, \Phi)$ be an IFZS with corresponding operator $T \in \mathscr{T}_{N}$. For $i^{*} \in\{1,2, \ldots, N\}$ define $E_{i^{*}}(T) \subset \mathscr{T}_{N}$, the " $\phi_{i^{*}}$-axis" of $\mathscr{T}_{N}$ at $T$, to be the section of $\mathscr{T}_{N}$ obtained by fixing the IFS maps $w_{i}, i \in\{1,2, \ldots, N\}$, and the grey-level maps $\phi_{i}, i \in\{1,2, \ldots, N\} \backslash\left\{i^{*}\right\}$, of $T$ and varying $\phi_{i} \in \mathscr{M}^{+}([0,1])$.

THEOREM 3.1. Let $T_{1} \in \mathscr{T}_{N}$, with corresponding IFZS ( $\left.\mathbf{w}, \Phi_{1}\right)$, where the IFS maps satisfy the following condition: for some $i^{*} \in\{1,2, \ldots, N\}$,

$$
d_{i^{*}} \equiv \sup _{x \in w_{i^{*}}(X)} \inf _{y \in w_{i^{*}}(X) \backslash \bigcup_{j \neq i}\left(w_{i^{*}}(X) \cap w_{j}(X)\right)} d(x, y)>0 .
$$

(In other words, $w_{i^{*}}(X)$ differs from $\bigcup_{j \neq i} w_{j}(X)$ by at least one point.) Also let $T_{2} \in$ $E_{i} \cdot\left(T_{1}\right)$, with corresponding IFZS $\left(\mathbf{w}, \Phi_{2}\right)$ such that $\phi_{1 i^{*}} \neq \phi_{2 i^{*}}$ and $\phi_{1 j}=\phi_{2 j}, j \neq i^{*}$. Then

$$
d_{C o n\left(\mathscr{F}^{*}(X)\right)}\left(T_{1}, T_{2}\right) \geq d_{i^{*}},
$$


independently of the $\phi_{k i}$ maps.

PROOF. Without loss of generality, let $i^{*}=1$. Using the notation of [3], define

$$
\beta_{1}(\alpha) \equiv \inf \left\{t \in[0,1]: \phi_{1,1} \geq \alpha\right\}, \quad \beta_{2}(\alpha) \equiv \inf \left\{t \in[0,1]: \phi_{2,1} \geq \alpha\right\}
$$

Assume, again without loss of generality, that $\phi_{1,1}\left(t^{*}\right)<\phi_{2,1}\left(t^{*}\right)$ for a certain $t^{*} \in$ $(0,1]$. Since the functions $\phi_{k i}$ are nondecreasing and right continuous, there exists an $\alpha^{*} \in[0,1]$ such that $\beta_{2}\left(\alpha^{*}\right)<\beta_{1}\left(\alpha^{*}\right)$; in fact, any $\alpha^{*} \in\left(\phi_{11}\left(t^{*}\right), \phi_{21}\left(t^{*}\right)\right)$ will work since $\beta_{2}\left(\alpha^{*}\right)<t^{*} \leq \beta_{1}\left(\alpha^{*}\right)$.

Let $x_{0} \in X$ be fixed so that $y_{0}=w_{1}\left(x_{0}\right)$ satisfies

$$
\sup _{y \in w_{1}(X) \backslash_{2 \leq j \leq N}\left(w_{1}(X) \cap w_{j}(X)\right)} d\left(y_{0}, y\right)=d_{1},
$$

and let $u_{0} \in \mathscr{F}^{*}(X)$ be defined as

$$
u_{0}(x)= \begin{cases}1, & x=x_{0} \\ \tilde{\beta}, & x \neq x_{0}\end{cases}
$$

where $\beta_{2}\left(\alpha^{*}\right)<\tilde{\beta}<\beta_{1}\left(\alpha^{*}\right)$. It follows that

$$
\begin{aligned}
{\left[\phi_{1,1} \circ u_{0}\right]^{\alpha^{*}} } & =\left[u_{0}\right]^{\beta_{1}\left(\alpha^{*}\right)}=\left\{x_{0}\right\}, \\
{\left[\phi_{2,1} \circ u_{0}\right]^{\alpha^{*}} } & =\left[u_{0}\right]^{\beta_{1}\left(\alpha^{*}\right)}=X, \\
{\left[\phi_{1 i} \circ u_{0}\right]^{\alpha^{*}} } & =\left[\phi_{2 i} \circ u_{0}\right]^{\alpha^{*}}, \quad i=2,3, \ldots, N .
\end{aligned}
$$

Then

$$
\begin{aligned}
& d_{\text {Con }\left(F^{*}(X)\right)}\left(T_{1}, T_{2}\right) \\
& =\sup _{u \in \mathscr{F}^{*}(X)} d_{\infty}\left(T_{1} u, T_{2} u\right) \\
& \geq d_{\infty}\left(T_{1} u_{0}, T_{2} u_{0}\right) \\
& =\sup _{0 \leq \alpha \leq 1} h\left(\left[T_{1} u_{0}\right]^{\alpha},\left[T_{2} u_{0}\right]^{\alpha}\right) \\
& \geq h\left(\left[T_{1} u_{0}\right]^{\alpha^{*}},\left[T_{2} u_{0}\right]^{\alpha^{*}}\right) \\
& =h\left(\bigcup_{1 \leq i \leq N} w_{i}\left(\left[\phi_{1 i} \circ u_{0}\right]^{\alpha^{*}}\right), \bigcup_{1 \leq j \leq N} w_{j}\left(\left[\phi_{2 j} \circ u_{0}\right]^{\alpha^{*}}\right)\right) \\
& =h\left(w_{1}\left(\left\{x_{0}\right\}\right) \cup\left(\underset{2 \leq i \leq N}{\cup} w_{i}\left(\left[\phi_{1 i} \circ u_{0}\right]^{\alpha^{*}}\right)\right), w_{1}(X) \cup\left(\underset{2 \leq j \leq N}{\cup} w_{j}\left(\left[\phi_{1 j} \circ u_{0}\right]^{\alpha^{*}}\right)\right)\right)
\end{aligned}
$$

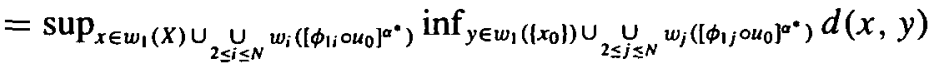

$$
\begin{aligned}
& =\sup _{x \in w_{1}(X)} \inf _{y \in w_{1}\left(( x _ { 0 } | ) \cup \bigcup _ { 2 \leq j \leq N } w _ { j } \left(\left[\phi_{1} \circ u_{0}\right]^{\left.\alpha^{*}\right)}\right.\right.} d(x, y) \\
& \geq \sup _{x \in w_{1}(x) \backslash \bigcup_{2 \leq j \leq N}\left(w_{1}(x) \cap w_{j}(X)\right)} \inf _{y \in w_{1}\left(\left|x_{0}\right|\right) \cup \bigcup_{2 \leq j \leq N} w_{j}(X)} d(x, y) \\
& =d_{1} \text {, }
\end{aligned}
$$

and the theorem is proved. 
This theorem shows that, in general, the $T$ operator is not continuous with respect to the grey level maps $\phi_{i}$. (The condition in (3.12) is not too restrictive. In fact, it omits only the rather special cases where each set $w_{i}(X)$ is contained in the union of all the other $w_{j}(X)$.) Unlike Proposition 3.4, however, we cannot guarantee, using the $d_{C o n\left(\mathscr{F}^{*}(X)\right)}$ metric, that the $d_{\infty}$ distance between two attractors $u_{1}$ and $u_{2}$ will be arbitrarily small by making the differences between the associated grey level vectors $\Phi_{1}$ and $\Phi_{2}$ sufficiently small.

As a final comment in this section, note that the lack of continuity does not contradict the property that the set of IFZS attractors is dense in the space $\left(\mathscr{F}^{*}(X), d_{\infty}\right)$ [5]. In this paper we are concerned only with IFZS having a fixed number, $N$, of IFS and grey level maps.

\section{Continuity of $u$ with respect to $w$ and $\Phi$}

In the previous section, the inequality in (3.3) shows that when $d_{\operatorname{Con}(Y)}$ is the "natural" metric in (3.10), then continuity of IFZS attractors $u$ on the space $Y=$ $\mathscr{F}^{*}(X)$ is uniform on the set of all contraction maps in $\left(\operatorname{Con}(Y), d_{C o n(Y)}\right)$. However, this uniform continuity is too restrictive: Theorem 3.1 shows that given an $\epsilon>0$ sufficiently small, the condition $d_{\operatorname{Con}\left(\mathscr{F}^{*}(X)\right)}\left(T_{1}, T_{2}\right)<\epsilon$ implies that $d_{\infty}\left(u_{1}, u_{2}\right)=0$. In this section, we show that a more practical continuity at each specific attractor $u \in \mathscr{F}^{*}(X)$ can be established, permitting a continuous variation of $u$ as both the w and the $\Phi$ components are perturbed. However, the perturbation of the $\Phi$ must be performed in a controlled manner: along the $\phi_{i}$-axes of $\mathscr{T}_{N}$ at the $T_{k}$ (Definition 3.1) together with restrictions as given in Theorem 4.2 below. Distance functions for the IFZS components will be defined as follows:

(a) For the IFS map vectors, we use the metric $d_{w}^{N}\left(\mathbf{w}_{1}, \mathbf{w}_{2}\right)$ defined in (3.5).

(b) For the grey level maps, first define the following distance function between two maps $\phi, \bar{\phi} \in \mathscr{M}^{+}([0,1])$ with respect to an element $u \in \mathscr{F}^{*}(X)$,

$$
d_{\phi}(\phi, \bar{\phi} ; u) \equiv \sup _{0 \leq \alpha \leq 1} h\left([\phi \circ u]^{\alpha},[\bar{\phi} \circ u]^{\alpha}\right),
$$

and the corresponding pseudometric for two grey level map vectors with respect to $u \in \mathscr{F}^{*}(X)$,

$$
d_{\phi}^{N}\left(\Phi_{1}, \Phi_{2} ; u\right) \equiv \max _{1 \leq i \leq N} d_{\phi}\left(\phi_{1 i}, \phi_{2 i} ; u\right) .
$$

THEOREM 4.1. Let $\left(\mathbf{w}_{k}, \Phi_{k}\right), k=1,2$ be two IFZS with corresponding contractive operators $T_{k} \in \mathscr{T}_{N}$ and attractors $u_{k}$, i.e., $T_{k} u_{k}=u_{k}, k=1,2$. Furthermore assume that $\phi_{1 i}(1)=\phi_{2 i}(1), i=1,2, \ldots, N$. Let $s_{1}$ be the contractivity factor of $\mathbf{w}_{1}$. Then

$$
d_{\infty}\left(u_{1}, u_{2}\right) \leq \frac{s_{1}}{1-s_{1}} d_{\phi}^{N}\left(\Phi_{1}, \Phi_{2} ; u_{1}\right)+\frac{1}{1-s_{1}} d_{w}^{N}\left(\mathbf{w}_{1}, \mathbf{w}_{2}\right) .
$$


PROOF. Let $T_{3} \in \mathscr{T}_{N}$ be the contractive operator corresponding to the "mixed" IFZS $\left(\mathbf{w}_{1}, \Phi_{2}\right)$. Then

$$
\begin{aligned}
d_{\infty}\left(u_{1}, u_{2}\right) & =d_{\infty}\left(T_{1} u_{1}, T_{2} u_{2}\right) \\
& \leq d_{\infty}\left(T_{1} u_{1}, T_{3} u_{1}\right)+\left(T_{3} u_{1}, T_{2} u_{2}\right) \\
& \leq d_{\infty}\left(T_{1} u_{1}, T_{3} u_{1}\right)+d_{\infty}\left(T_{3} u_{1}, T_{3} u_{2}\right)+d_{\infty}\left(T_{3} u_{2}, T_{2} u_{2}\right) \\
& \leq d_{\infty}\left(T_{1} u_{1}, T_{3} u_{1}\right)+s_{1} d_{\infty}\left(u_{1}, u_{2}\right)+d_{\infty}\left(T_{3} u_{2}, T_{2} u_{2}\right) .
\end{aligned}
$$

Hence

$$
\left(1-s_{1}\right) d_{\infty}\left(u_{1}, u_{2}\right) \leq d_{\infty}\left(T_{1} u_{1}, T_{3} u_{1}\right)+d_{\infty}\left(T_{3} u_{2}, T_{2} u_{2}\right)
$$

We now examine the two distances on the right side of (4.5). Since

$$
\begin{aligned}
h\left(\bigcup_{i=1}^{N} w_{1 i}\left(\left[\phi_{1 i} \circ u_{1}\right]^{\alpha}\right)\right. & \left., \bigcup_{j=1}^{N} w_{1 j}\left(\left[\phi_{2 j} \circ u_{1}\right]^{\alpha}\right)\right) \\
& \leq \max _{1 \leq i \leq N} h\left(w_{1 i}\left(\left[\phi_{1 i} \circ u_{1}\right]^{\alpha}\right), w_{1 i}\left(\left[\phi_{2 i} \circ u_{1}\right]^{\alpha}\right)\right) \\
& \leq s_{1} \max _{1 \leq i \leq N} h\left(\left[\phi_{1 i} \circ u_{1}\right]^{\alpha},\left[\phi_{2 i} \circ u_{1}\right]^{\alpha}\right),
\end{aligned}
$$

it follows that

$$
d_{\infty}\left(T_{1} u_{1}, T_{3} u_{1}\right) \leq s_{1} d_{\phi}^{N}\left(\Phi_{1}, \Phi_{2} ; u_{1}\right) .
$$

(Note that the assumption $\phi_{1 i}(1)=\phi_{2 i}(1), i=1,2, \ldots, N$ is necessary to guarantee that the level sets $\left[\phi_{1 i} \circ u_{1}\right]^{\alpha}$ and $\left[\phi_{2 i} \circ u_{1}\right]^{\alpha}$ in (4.6) exist for the same range of $\alpha$-values.) Moreover,

$$
\begin{aligned}
h\left(\bigcup_{i=1}^{N} w_{1 i}\left(\left[\phi_{2 i} \circ u_{2}\right]^{\alpha}\right)\right. & \left., \bigcup_{j=1}^{N} w_{2 j}\left(\left[\phi_{2 j} \circ u_{2}\right]^{\alpha}\right)\right) \\
& \leq \max _{1 \leq i \leq N} h\left(w_{1 i}\left(\left[\phi_{2 i} \circ u_{2}\right]^{\alpha}\right), w_{2 i}\left(\left[\phi_{2 i} \circ u_{2}\right]^{\alpha}\right)\right) \\
& \leq d_{w}^{N}\left(\mathbf{w}_{1}, \mathbf{w}_{2}\right) .
\end{aligned}
$$

Thus, (4.5), (4.7) and (4.8) imply that

$$
\left(1-s_{1}\right) d_{\infty}\left(u_{1}, u_{2}\right) \leq s_{1} d_{\phi}^{N}\left(\Phi_{1}, \Phi_{2} ; u_{1}\right)+d_{w}^{N}\left(\mathbf{w}_{1}, \mathbf{w}_{2}\right)
$$

and the desired result follows.

The following lemmas will be useful for the proof of Theorem 4.2, the major result of this section.

LeMMA 4.1. Given $A_{i}, B_{i}, C_{i} \in \mathscr{H}(X), i=1,2$, such that

$$
A_{1} \subseteq B_{1} \subseteq C_{1}, \quad A_{2} \subseteq B_{2} \subseteq C_{2},
$$

then

$$
h\left(B_{1}, B_{2}\right) \leq \max \left\{h\left(A_{2}, C_{1}\right), h\left(A_{1}, C_{2}\right)\right\} .
$$


PROOF.

$$
\begin{aligned}
D\left(B_{1}, B_{2}\right) & =\sup _{x \in B_{1}} \inf y \in B_{2} \\
& \leq \sup _{x \in C_{1}} d\left(x \in A_{2}\right. \\
& =D\left(C_{1}, A_{2}\right) \\
& \leq h\left(C_{1}, A_{2}\right) .
\end{aligned}
$$

Similarly, $D\left(B_{2}, B_{1}\right) \leq D\left(C_{2}, A_{1}\right) \leq h\left(C_{2}, A_{1}\right)$. The result in (4.12) then follows.

LEMMA 4.2. If $u \in \mathscr{F}^{*}(X)$, then

(i) $[u]^{\alpha}$ is closed for all $\alpha \in[0,1]$,

(ii) If $\beta \uparrow \beta^{*}$, then

$$
[u]^{\beta} \rightarrow \underset{\beta<\beta^{*}}{\cap}[u]^{\beta}=[u]^{\beta^{*}}
$$

in Hausdorff metric.

Property (i) follows from the upper semicontinuity of $u \in \mathscr{F}^{*}(X)$. Property (ii) was proved in [4].

The following lemma establishes the existence of $\phi$ maps in a restricted neighbourhood of a given $\bar{\phi} \in \mathscr{M}^{+}([0,1])$ in terms of the distance function $d_{\phi}(\phi, \bar{\phi} ; u)$ introduced in (4.2) and defined with respect to a given $u \in \mathscr{F}^{*}(X)$. These $\phi$ maps are obtained by suitably perturbing $\bar{\phi}$, which is nonconstant on $[0,1]$. (Recall the definition of $\mathscr{M}^{+}([0,1])$.)

LemMa 4.3. Let $u \in \mathscr{F}^{*}(X)$ and $\bar{\phi} \in \mathscr{M}^{+}([0,1])$. Assume that for some $\alpha^{*}>0$ and some $t^{*} \in(0,1), \bar{\phi}\left(t^{*}\right)=\alpha^{*}$. Let

$$
\bar{\beta}^{*}:=\bar{\beta}\left(\alpha^{*}\right) \equiv \inf \left\{t \in[0,1]: \bar{\phi}(t) \geq \alpha^{*}\right\} .
$$

Thenfor every $\epsilon>0$, there exists an $\eta^{*}, 0<\eta^{*}<\bar{\beta}^{*}$, such that for all $\phi \in \mathscr{M}^{+}([0,1])$ satisfying the following properties:

(a) $\phi(t)=\bar{\phi}(t)$ for $0 \leq t<\bar{\beta}^{*}-\eta^{*}$ and $\bar{\beta}^{*} \leq t \leq 1$,

(b) $\phi(t) \geq \bar{\phi}(t)$ for $\bar{\beta}^{*}-\eta^{*} \leq t \leq \bar{\beta}^{*}$,

we have

$$
d_{\phi}(\bar{\phi}, \phi ; u)<\epsilon
$$

Proof. Since $\bar{\phi}$ is right continuous and $\bar{\phi}(0)=0$, it follows that $\bar{\beta}^{*}>0$. Property (ii) of Lemma 4.2 implies that for every $\epsilon>0$ there exists an $\eta^{*}>0, \bar{\beta}^{*}-\eta^{*}>0$ such that

$$
h\left([u]^{\beta},[u]^{\bar{\beta}}\right)<\epsilon, \quad \forall \beta \in\left[\bar{\beta}^{*}-\eta^{*}, \bar{\beta}^{*}\right] .
$$


Now let $\phi \in \mathscr{M}^{+}([0,1]) \backslash\{\bar{\phi}\}$ be a function that satisfies (a) and (b) above. If $0 \leq \alpha_{1} \leq \alpha \leq \alpha_{2} \leq 1$, then for any $\phi, \bar{\phi} \in \mathscr{M}^{+}([0,1])$, with

$$
\beta(\alpha) \equiv \inf \{t \in[0,1]: \phi(t)>\alpha\}
$$

and

$$
\bar{\beta}(\alpha) \equiv \inf \{t \in[0,1]: \bar{\phi}(t)>\alpha\},
$$

we have the following relations:

$$
\begin{aligned}
& {[\bar{\phi} \circ u]^{\alpha_{2}} \subseteq[\bar{\phi} \circ u]^{\alpha} \subseteq[\bar{\phi} \circ u]^{\alpha_{1}} \text {, i.e., }[u]^{\bar{\beta}\left(\alpha_{2}\right)} \subseteq[u]^{\bar{\beta}(\alpha)} \subseteq[u]^{\bar{\beta}\left(\alpha_{1}\right)} \text {, }} \\
& {[\phi \circ u]^{\alpha_{2}} \subseteq[\phi \circ u]^{\alpha} \subseteq[\phi \circ u]^{\alpha_{1}} \text {, i.e., }[u]^{\beta\left(\alpha_{2}\right)} \subseteq[u]^{\beta(\alpha)} \subseteq[u]^{\beta\left(\alpha_{1}\right)} \text {. }}
\end{aligned}
$$

If, in particular,

$$
\alpha_{2}=\alpha^{*}, \quad \alpha_{1}=\phi\left(\bar{\beta}^{*}-\eta^{*}\right),
$$

and $\phi$ satisfies the conditions (a) - (c) above, then

$$
\begin{aligned}
& A_{1} \equiv[\bar{\phi} \circ u]^{\alpha_{2}}=[\phi \circ u]^{\alpha_{2}} \equiv A_{2}, \\
& C_{1} \equiv[\bar{\phi} \circ u]^{\alpha_{1}} \subseteq[\phi \circ u]^{\alpha_{1}} \equiv C_{2} .
\end{aligned}
$$

Letting $B_{1}=[\bar{\phi} \circ u]^{\alpha}$ and $B_{2}=[\phi \circ u]^{\alpha}$, we have, from Lemma 4.1 and the fact that $C_{1} \subseteq C_{2}$,

$$
h\left([\bar{\phi} \circ u]^{\alpha},[\phi \circ u]^{\alpha}\right) \leq h\left([\bar{\phi} \circ u]^{\alpha_{2}},[\phi \circ u]^{\alpha_{1}}\right)=h\left([u]^{\bar{\beta}^{*}},[u]^{\bar{\beta}^{*}-\eta^{*}}\right)<\epsilon .
$$

On the other hand, by construction, $h\left([\bar{\phi} \circ u]^{\alpha},[\phi \circ u]^{\alpha}\right)=0$ for $\alpha \in\left[0, \bar{\phi}\left(\bar{\beta}^{*}-\right.\right.$ $\left.\left.\eta^{*}\right)\right) \cup\left(\bar{\phi}\left(\bar{\beta}^{*}\right), 1\right]$. (Note that $\bar{\phi}\left(\bar{\beta}^{*}-\eta^{*}\right)<\bar{\phi}\left(\bar{\beta}^{*}\right)$, from the definition of $\bar{\beta}^{*}$ and the right continuity of $\bar{\phi}$.) Hence

$$
d_{\phi}(\bar{\phi}, \phi ; u)=\sup _{0 \leq \alpha \leq 1} h\left([\bar{\phi} \circ u]^{\alpha},[\phi \circ u]^{\alpha}\right)<\epsilon
$$

and the proof is complete.

We now come to the major result regarding continuity of IFZS attractor functions.

THEOREM 4.2. Given an IFZS $\left(\mathbf{w}_{1}, \Phi_{1}\right)$ with corresponding contractive operator $T_{1} \in$ $\mathscr{T}_{N}$ and fixed point $u_{1} \in \mathscr{F}^{*}(X)$, then for every $\epsilon>0$ and every $\epsilon_{1}, \epsilon_{2}>0$ such that (see Theorem 4.1)

$$
\frac{s_{1}}{1-s_{1}} \epsilon_{2}+\frac{1}{1-s_{1}} \epsilon_{1}<\epsilon,
$$

there exist the following: 
(1) an IFS $\mathbf{w}_{2} \neq \mathbf{w}_{1}$ for which

$$
d_{w}^{N}\left(\mathbf{w}_{1}, \mathbf{w}_{2}\right)<\epsilon_{1},
$$

(2) a set of grey level maps $\Phi_{2} \neq \Phi_{1}$ such that

$$
d_{\phi}^{N}\left(\Phi_{1}, \Phi_{2} ; u_{1}\right)<\epsilon_{2}
$$

As such, the operator $T_{2} \in \mathscr{T}_{N}$ corresponding to $\left(\mathbf{w}_{2}, \Phi_{2}\right)$ has a fixed point $u_{2} \in$ $\mathscr{F}^{*}(X)$, such that

$$
d_{\infty}\left(u_{1}, u_{2}\right)<\epsilon .
$$

Moreover, for every $\epsilon>0$, there exist $\delta_{1}>0\left(\delta_{1}=\epsilon_{1}\left(1-s_{1}\right)^{-1}\right)$ and $\delta_{2}>0$ $\left(\delta_{2}=s_{1} \epsilon_{2}\left(1-s_{1}\right)^{-1}\right)$ such that $d_{w}^{N}\left(\mathbf{w}_{1}, \mathbf{w}_{2}\right)<\delta_{1}$ and $d_{\phi}^{N}\left(\Phi_{1}, \Phi_{2} ; u_{1}\right)<\delta_{2}$ imply (4.18).

PROOF. Regarding (1), the existence of an IFS $\mathbf{w}_{2}$ satisfying the inequality in (1) is straightforward, see [1]. Regarding (2), recall that for each $i^{*} \in\{1,2, \ldots, N\}$ there exists at least one value $t^{*} \in[0,1]$, such that $\phi_{1, i^{*}}\left(t^{*}\right)>0$. Then, from Lemma 4.3, the following set $\Phi_{2}$ of grey level maps satisfies condition (2):

$$
\begin{aligned}
\phi_{2, i} & =\phi_{1, i}, \quad i \in\{1,2, \ldots, N\} \backslash\left\{i^{*}\right\}, \\
\phi_{2, i^{*}}(t) & =\phi_{1, i^{*}}(t), \quad t \in[0,1] \backslash\left[t^{*}-\eta^{*}, t^{*}\right), \\
\phi_{2, i^{*}}(t) & \geq \phi_{1, i^{*}}(t), \quad t \in\left[t^{*}-\eta^{*}, t^{*}\right),
\end{aligned}
$$

where $\eta^{*}$ is such that (see Lemma 4.3)

$$
d_{\phi}\left(\phi_{1, i^{*}}, \phi_{2, i} ; u_{1}\right)<\epsilon_{2}
$$

Since

$$
d_{\phi}^{N}\left(\Phi_{1}, \Phi_{2} ; u_{1}\right)=\sup _{1 \leq i \leq N} d\left(\phi_{2, i}, \phi_{1, i} ; u_{1}\right)=d\left(\phi_{2, i^{*}}, \phi_{1, i^{*}} ; u_{1}\right),
$$

(4.18) follows from Theorem 4.1 and the proof is complete.

Note that the conditions $\phi_{1 i}(1)=\phi_{2 i}(1), i=1,2, \ldots, N$, assumed to hold in Theorem 4.1, are essential for the existence of a $\Phi_{2}$ such that (4.17) holds for a given $\epsilon_{2}>0$ and $u_{1}$. The last part of Theorem 4.2 (continuity of $u$ at $u_{1}$ ) is a direct consequence of Theorem 4.1.

\section{Discussion}

For practical purposes, e.g., animation or the inverse problem of fractal construction, it is desirable that an IFZS attractor $u_{k} \in \mathscr{F}^{*}(X)$ may be varied in a continuous 
fashion by varying the IFS maps $w_{k i}$ and the grey level maps $\phi_{k i}$. The IFZS attractors $u_{k}$ are trivially continuous with respect to the operators $T_{k}$ in the $d_{C o n_{T}^{N}\left(F^{*}(X)\right)}$ metric. However, we have shown that the $T_{k}$ may be discontinuous along the sections $E_{i}\left(T_{k}\right)$ obtained by varying the $\phi_{k i}$ one at a time. These sections are naturally important in the "fine-tuning" of grey level distributions on $u_{k}$. (We should mention that our use of the $E_{i}\left(T_{k}\right)$ sections in the above analysis is in no way restrictive: a standard triangular inequality argument clearly shows that a continuous variation in the grey level vector $\Phi=\left(\phi_{1}, \phi_{2}, \ldots, \phi_{N}\right)$ with respect to the $d_{\phi}^{N}$ metric can be achieved by moving along the $E_{i}\left(T_{k}\right)$ sections, i.e., varying the $\phi_{k i}$ one map at a time.)

In order to remedy this situation, a "local" metric for the grey level maps with respect to an element $u \in \mathscr{F}^{*}(X)$ was constructed. The continuity of attractors $u_{k}$ with respect to the $\phi_{k i}$ maps was then established, subject to some restrictions on their variations (Section 4). These restrictions are not too serious: the perturbation method outlined in Theorem 4.2 still allows freedom in the amount of variation in the $\phi_{k i}$. In fact, any additional regularity in the $\phi_{k i}$ maps, e.g., bijectivity, will allow even more freedom in the variation. However, the restriction that the values $\phi_{k i}(1)$ be fixed is essential. It can easily be shown (along the lines of Theorem 3.1) that any $\delta$-variation in this value produces an attractor $\bar{u}_{k}$ whose $d_{\infty}$ distance from $u_{k}$ is bounded away from zero independently of $\delta$.

An even greater flexibility in controlling the $u_{k}$ attractors could be accomplished if the grey level maps $\phi_{k i}$ were functions of the base space variable $x \in X$, i.e., $\phi: X \times[0,1] \rightarrow[0,1]$. As well, it may be advantageous to consider operators other than the $T$ operator of (2.7) and, consequently, other metrics on $\mathscr{F}^{*}(X)$ (in order to preserve the contractivity of $T$ or to possibly guarantee uniform continuity of the attractors). Recently, in fact, we have succeeded in modifying the original IFZS method [8]. One of the modifications involves a new distance function which, in special cases, becomes the $L^{1}(X)$ distance with respect to a measure $\mu$ on $X$. The attractors of this new IFS-type method on $L^{1}(X)$ are then trivially continuous with respect to the grey-level maps $\phi_{i}$. This new approach has been very effective in solving the inverse problem of function approximation and image representation.

\section{Acknowledgements}

This research was supported in part by grants (BF, ERV) from the Natural Sciences and Engineering Research Council of Canada, which are gratefully acknowledged. 


\section{Appendix A: IFS with probabilities and invariant measures}

An $N$-map contractive IFS on $X$ with probabilities (IFSP), (w, $\mathbf{p})$, is a set of $N$ contraction maps $\mathbf{w}=\left(w_{1}, w_{2}, \ldots, w_{N}\right), w_{i} \in \operatorname{Con}(X)$ with associated probabilities $\mathbf{p}=\left(p_{1}, p_{2}, \ldots, p_{N}\right), p_{i} \geq 0, \sum_{i=1}^{N} p_{i}=1$. The uniqueness of the attractor $A$ defined by the set $w$ was discussed in Section 2.

Now let $\mathscr{M}(X)$ denote the set of probability measures on $\mathscr{B}(X)$, the $\sigma$-algebra of Borel subsets of $X$. Consider the following metric on $\mathscr{M}(X)$,

$$
d_{H}(\mu, v)=\sup _{f \in \operatorname{Lip}(X)}\left[\int f d \mu-\int f d \nu\right] \quad \mu, \nu \in \mathscr{M}(X),
$$

where

$$
\operatorname{Lip}(X)=\{f: X \rightarrow \mathbf{R},|f(x)-f(y)| \leq d(x, y), x, y \in X\} .
$$

The $d_{H}$ metric has often been called the Hutchinson metric due to its use in [9]. Note that $\left(\mathscr{M}(X), d_{H}\right)$ is a complete metric space.

Given an IFSP (w, p), define a "Markov operator" $M: \mathscr{M}(X) \rightarrow \mathscr{M}(X)$ as follows: for $v \in \mathscr{M}(X)$, let

$$
M \nu=\sum_{i=1}^{N} p_{i} \nu \circ w_{i}^{-1}
$$

Then there exists a unique measure $\mu \in \mathscr{M}(X)$, the invariant measure of the IFSP (w, p), for which $M \mu=\mu . M$ is a contraction mapping on $\left(\mathscr{M}(X), d_{H}\right)$, i.e., for $\mu, \nu \in \mathscr{M}(X)[9]$,

$$
d_{H}(M \mu, M \nu)<s d_{H}(\mu, \nu),
$$

where $s$ is the contractivity factor of $\mathbf{w}$.

EXAMPLES. Return to the 2-map IFS on $[0,1]$ given earlier in Section 2. (i) When $s=1 / 2$ and $p_{1}=p_{2}=1 / 2$, then $\mu$ is Lebesgue measure on $[0,1]$. (ii) When $s=1 / 3$ and $p_{1}=p_{2}=1 / 2$, then $\mu$ is the uniform Cantor-Lebesgue measure on the ternary Cantor set on $[0,1]$.

\section{References}

[1] M. F. Barnsley, Fractals everywhere (Academic Press, New York, 1988).

[2] M. F. Barnsley, Fractal image compression (A. K. Peters, Wellesley, MA, 1993).

[3] M. F. Barnsley, D. Hardin, V. Ervin and J. Lancaster, "Solution of an inverse problem for fractals and other sets”, Proc. Nat. Acad. Sci. USA 83 (1986) 1975-1977. 
[4] C. A. Cabrelli, B. Forte, U. M. Molter and E. R. Vrscay, "Iterated fuzzy set systems: a new approach to the inverse problem for fractals and other sets", J. Math. Anal. Appl. 171 (1992) 79-100.

[5] C. A. Cabrelli and U. M. Molter, "Density of fuzzy set attractors: a step towards the solution of the inverse problem for fractals and other sets", Proc. NATO Advanced Study Institute on Probabilistic and Stochastic Methods in Analysis with Applications (July 1991).

[6] P. Centore and E. R. Vrscay, "Continuity of attractors and invariant measures for iterated function systems", Can. Math. Bull. (1993), to appear.

[7] P. Diamond and P. Kloeden, "Metric spaces of fuzzy sets", Fuzzy Sets and Systems 35 (1990) 241-249.

[8] B. Forte and E. R. Vrscay, "Approximation of functions and images in $L^{1}(X)$ using iterated function systems", in preparation.

[9] J. Hutchinson, "Fractals and self-similarity", Indiana Univ. J. Math. 30 (1981) 713-747.

[10] A. Jacquin, "A fractal theory of iterated Markov operators with applications to digital image coding", Ph. D. Thesis, Georgia Institute of Technology, 1989.

[11] E. R. Vrscay, "Iterated function systems: theory, application, and the inverse problem", in Proc. NATO Advanced Study Institute on Fractal Geometry and Analysis (eds. J. Bélair and S. Dubuc), NATO ASI Series C 346, (Kluwer, Dordrecht, The Netherlands, 1991), 405-468. 